Title : will be set by the publisher

Editors : will be set by the publisher

EAS Publications Series, Vol. ?, 2018

\title{
OBSERVATIONS OF PROTOSTELLAR OUTFLOW FEEDBACK IN CLUSTERED STAR FORMATION
}

\author{
Fumitaka Nakamura $^{1}$
}

\begin{abstract}
We discuss the role of protostellar outflow feedback in clustered star formation using the observational data of recent molecular outflow surveys toward nearby cluster-forming clumps. We found that for almost all clumps, the outflow momentum injection rate is significantly larger than the turbulence dissipation rate. Therefore, the outflow feedback is likely to maintain supersonic turbulence in the clumps. For less massive clumps such as B59, L1551, and L1641N, the outflow kinetic energy is comparable to the clump gravitational energy. In such clumps, the outflow feedback probably affects significantly the clump dynamics. On the other hand, for clumps with masses larger than about $200 \mathrm{M}_{\odot}$, the outflow kinetic energy is significantly smaller than the clump gravitational energy. Since the majority of stars form in such clumps, we conclude that outflow feedback cannot destroy the whole parent clump. These characteristics of the outflow feedback support the scenario of slow star formation.
\end{abstract}

\section{Introduction}

Most stars form in clustered environments (Lada 2010). Radio and infrared ob' servations have revealed that star clusters form in parsec-scale dense molecular clumps with masses of the order of $10^{2}-10^{3} \mathrm{M}_{\odot}$. In such clumps, stellar feedback from forming stars shapes their internal structure and affects the formation of next-generation stars. Therefore, understanding the role of stellar feedback in the process of cluster formation is a central problem of star formation study.

There are several types of stellar feedback such as outflows, winds, and radiation (Bally 2010, Krumholz et al. 2014). Among them, some theoretical studies suggest that the radiation pressure from high-mass stars is the most dominant feedback mechanism in the presence of high-mass stars. However, in the process of high-mass star formation, the outflow feedback from low-mass stars may play a dominant role in regulating star formation before high-mass stars are formed.

${ }^{1}$ National Astronomical Observatory of Japan, 2-21-1 Osawa, Mitaka, 181-8588, Japan

(c) EDP Sciences 2018

DOI: (will be inserted later) 
Title : will be set by the publisher

In other words, the outflow feedback can control the formation of high-mass stars by injecting momentum and energy in the surrounding gas. In addition, there are many cluster-forming regions where no high-mass stars form. In such regions, outflow feedback should play a dominant role in regulating star formation. In this contribution, we focus on the protostellar outflow feedback and discuss its role on the clump dynamics on the basis of observations.

Protostellar outflows have been shown theoretically to be capable of maintaining supersonic turbulence in cluster-forming clumps and keeping the star formation rate per free-fall time as low as a few percent (Nakamura \& Li 2007). However, its exact role in clustered star formation remains controversial. Two main scenarios have been proposed for the role of outflow feedback in clustered star formation. In the first scenario, the outflow feedback is envisioned to destroy the cluster-forming clump as a whole, which terminates further star formation. In this case, star formation should be rapid and brief (e.g., Mac Low \& Klessen 2004). On the other hand, in the second scenario, the outflow feedback is envisioned to play the role of maintaining the internal turbulent motions. In this scenario, star formation should be slow and can last for several free-fall times or longer (e.g., Tan et al. 2006). Below we constrain these theoretical models using the observational results of the protostellar outflow feedback.

\section{Data: Protostellar Outflows}

\begin{tabular}{lccc|l|}
\hline Clumps & $\begin{array}{c}\text { distance } \\
(\mathrm{pc})\end{array}$ & $\begin{array}{c}\text { Mass } \\
\left(\mathrm{M}_{\odot}\right)\end{array}$ & $\begin{array}{c}\text { Radiius } \\
(\mathrm{pc})\end{array}$ & References \\
\hline B59 & 130 & 30 & 0.3 & Duarte-Cabral et al. (2012) \\
L1551 & 140 & 110 & 1.0 & Stojimirovic et al. (2006) \\
L1641N & 400 & 210 & 0.55 & Nakamura et al. (2012) \\
Serpens Main & 415 & 535 & 0.73 & Sugitani et al. (2010) \\
Serpens South & 415 & 232 & 0.2 & Nakamura et al. (2011b) \\
$\rho$ Oph & 125 & 883 & 0.8 & Nakamura et al. (2011a) \\
IC 348 & 250 & 620 & 0.9 & Arce et al. (2010) \\
NGC 1333 & 250 & 1100 & 2.0 & Arce et al. (2010) \\
NGC 2264-C & 800 & 2300 & 0.7 & Maury et al. (2009) \\
\hline
\end{tabular}

Table 1. Nearby cluster-forming clumps. We note that the clump masses are estimated by ${ }^{13} \mathrm{CO}$ data except for Serpens South, for which the Herschel data are used because of severe absorption of the ${ }^{13} \mathrm{CO}$ emission.

The outflowing gas from protostars accelerates entrained gas to large velocities. Such components are observed as molecular outflows and such molecular outflows are important to inject momentum in the surrounding gas. The $\mathrm{CO}$ lines such as the $J=1-0, J=2-1$, and $J=3-2$ transitions are excellent tracers to measure the parameters of molecular outflows. In Table 1, we summarize some properties of nearby cluster-forming clumps toward which $\mathrm{CO}$ outflow surveys were carried out. In the next section, we discuss how $\mathrm{CO}$ outflows affect the parent clumps 
using the observational results presented in the references listed in Table 1 .

Outflow feedback has two different roles. One is negative effect, slowing down or terminating star formation by injecting momentum and energy. In this case, the mean densities of regions decrease by feedback. Another role is positive effect, triggering future star formation by dynamically compressing the surroundings. In this case, the local densities increase by compression. For outflow feedback, the negative effect is likely to be more important than positive one because the outflow feedback happens inside the star-forming regions, dispersing gas outwards. Therefore, in the following we focus on the negative effect of the outflow feedback. In the next section, we address the following two important questions of star formation using the observational data.

(1) Can protostellar outflow feedback maintain supersonic turbulence in clustered environment?

(2) Can protostellar outflow feedback directly destroy the parent clumps?

\begin{tabular}{lll}
\hline Model & $(1)$ & $(2)$ \\
\hline Rapid Star Formation & Yes/No & Yes \\
Slow Star Formation & Yes & No \\
\hline
\end{tabular}

Table 2. Rapid vs. slow star formation models.

The two models of star formation give the different answers to these questions. In Table 2. we summarize the predicted answers of these two questions for the two star formation models.

\section{Results}

First, using the observational data of the outflow surveys listed in Table 1, we calculated some physical parameters of the outflow feedback in Table 3.

Here, we assumed that outflow gas is optically-thin and that the inclination angles of all the identified outflows are fixed to be the mean value of 57.3 degree. However, low-velocity wings sometimes become optically thick. Therefore, the assumption of the optically-thin leads to underestimation of the outflow mass by a factor of 10 or more. However, we note that we may underestimate momentum and energy only by a factor of a few because the optically-thick gas have low-velocity, and have less momentum and energy than high-velocity components.

\subsection{Can outflow feedback maintain supersonic turbulence?}

To answer this question, we compare the turbulence dissipation rate $\left(d P_{\text {turb }} / d t\right)$ and outflow momentum injection rates $\left(d P_{\text {out }} / d t\right)$ towards target clumps. In previous studies, the energy is used for the analysis. However, because the outflow feedback is the momentum feedback (Krumholz et al. 2014), we use momentum instead of energy. The dissipation rate of turbulence is defined as the total cloud 
Title : will be set by the publisher

\begin{tabular}{lccclll}
\hline Clumps & $\alpha$ & $\sigma$ & $d P_{\text {turb }} / d t$ & $d P_{\text {out }} / d t$ & $E_{\text {out }}$ & $E_{\text {grav }}$ \\
\hline B59 & 1.1 & 0.4 & $1.0 \times 10^{-5}$ & $8.5 \times 10^{-5}$ & 4 & 13 \\
L1551 & 1.3 & 0.45 & $1.8 \times 10^{-5}$ & $6.3 \times 10^{-4}$ & 130 & 52 \\
L1641N & 1.0 & 0.74 & $1.3 \times 10^{-4}$ & $1.3 \times 10^{-3}$ & 273 & 581 \\
Serpens Main & 0.7 & 0.85 & $3.4 \times 10^{-4}$ & $2.5 \times 10^{-3}$ & 445 & 1686 \\
Serpens South & 0.2 & 0.53 & $2.1 \times 10^{-4}$ & $6.5 \times 10^{-4}$ & 165 & 1157 \\
$\rho$ Oph & 0.2 & 0.64 & $2.9 \times 10^{-4}$ & $1.2 \times 10^{-4}$ & 61 & 4191 \\
IC 348 & 0.6 & 0.76 & $2.5 \times 10^{-4}$ & $4.7 \times 10^{-4}$ & 26 & 1837 \\
NGC 1333 & 1.1 & 0.93 & $3.0 \times 10^{-4}$ & $1.1 \times 10^{-3}$ & 119 & 2602 \\
NGC 2264-C & 0.6 & 1.7 & $5.5 \times 10^{-4}$ & $1.7 \times 10^{-3}$ & 50 & 27652 \\
\hline
\end{tabular}

Table 3. Some physical parameters of the outflow feedback for nearby cluster-forming clumps. $\alpha$ is the virial ratio, $\sigma$ is $1 \mathrm{D}$ velocity dispersion $\left(\mathrm{km} \mathrm{s}^{-1}\right), d P_{\mathrm{turb}} / d t$ is the dissipation rate of turbulence $\left(M_{\odot} \mathrm{km} \mathrm{s}^{-1} \mathrm{yr}^{-1}\right), d P_{\text {out }} / d t$ is the outflow momentum injection rate $\left(M_{\odot} \mathrm{km} \mathrm{s}^{-1} \mathrm{yr}^{-1}\right), E_{\text {out }}$ is the outflow kinetic energy $\left(M_{\odot} \mathrm{km}^{2} \mathrm{~s}^{-2}\right), E_{\text {grav }}$ is the clump gravitational energy $\left(M_{\odot} \mathrm{km}^{2} \mathrm{~s}^{-2}\right)$.

momentum divided by turbulence crossing time $\left(d P_{\text {turb }} / d t=0.21 M_{\mathrm{cl}} \sigma_{3 \mathrm{D}} / t_{\mathrm{diss}}\right)$. The numerical factor of 0.21 is determined from comparison with turbulence simulations. The outflow momentum injection rate is defined by the outflow momentum divided by the outflow dynamical time.

In Figure 1, we show the ratio between momentum injection rate and dissipation rate as a function of clump mass. The momentum injection rates are larger than the dissipation rates for all clumps except IC 348. Thus, we conclude that the outflow feedback has enough momentum to maintain supersonic turbulence in these clumps.

\subsection{Can outflow feedback directly destroy the parent clump?}

To answer this question, we adopt the virial theorem, $d^{2} I / d t^{2}=2 E_{\mathrm{cl}}-E_{\text {grav }}$. First, we list the virial ratios $\left(\alpha=2 E_{\mathrm{cl}} / E_{\text {grav }}\right)$ of the clumps in Table 3 . Almost all clumps are close to virial equilibrium within a factor of a few. In Figure 2 , we show the ratios of $2 E_{\text {out }}$ and $E_{\text {grav }}$ as a function of clump mass. Figure 2 indicates that for less massive clumps (B59, L1551, L1641N, < $200 \mathrm{M}_{\odot}$ ), the outflow energy is comparable to the gravitational energy. The dynamics of such clumps may be significantly influenced by the outflow feedback. On the other hand, for more massive clumps $\left(>200 \mathrm{M}_{\odot}\right)$, the outflow kinetic energy appears to be significantly smaller than the gravitational energy. In other words, the outflow feedback may not directly destroy the whole clumps. But, a fraction of gas may be dispersed by outflows. This gentle ejection of gas may lead to clump dispersal eventually.

\section{Slow vs. Rapid Star Formation}

From the results presented in the previous section, we found that the outflow feedback can maintain supersonic turbulence in the nearby cluster-forming clumps. In addition, the outflow kinetic energy is significantly smaller than the clump 


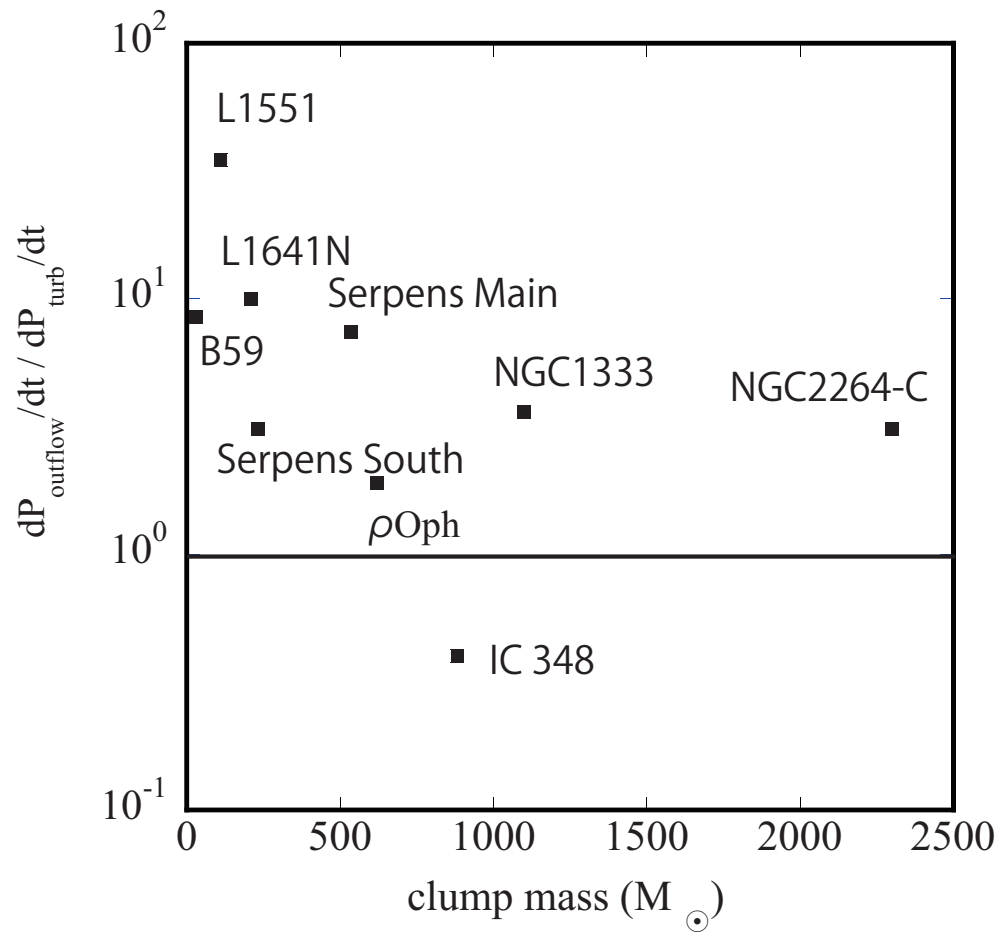

Fig. 1. Ratios of the momentum injection to the turbulence dissipation rates of nearby cluster-forming clumps as a function of clump mass. Our result seems to be inconsistent with that of Maury et al. (2006) for NGC 2264-C. The difference comes from the fact that they use the energy dissipation rate, instead of the momentum dissipation rate. In addition, they omitted the numerical factor of 0.21 to estimate the dissipation rate of turbulence (see Nakamura \& Li 2014).

gravitational energy except for the three least massive clumps, B59, L1551, and L1641N. Therefore, we conclude that the outflow feedback is not enough to disperse the whole clump at least for the clumps with masses greater than $200 \mathrm{M}_{\odot}$. Since the majority of stars form in such clumps, we conclude that the observations of the outflow feedback support the slow star formation.

\section{References}

Arce, H. G., Borkin, M. A., Goodman, A. A., Pineda, J. E., \& Halle, M. W. 2010, ApJ, 715,1170

Bally, J. 2010, in Computational Star Formation, Proceedings of the International Astronomical Union, IAU Symposium, 270, p.247

Duarte-Cabral, A., Chrysostomou, A., Peretto, N., et al. 2012, A\&A, 543, 140 


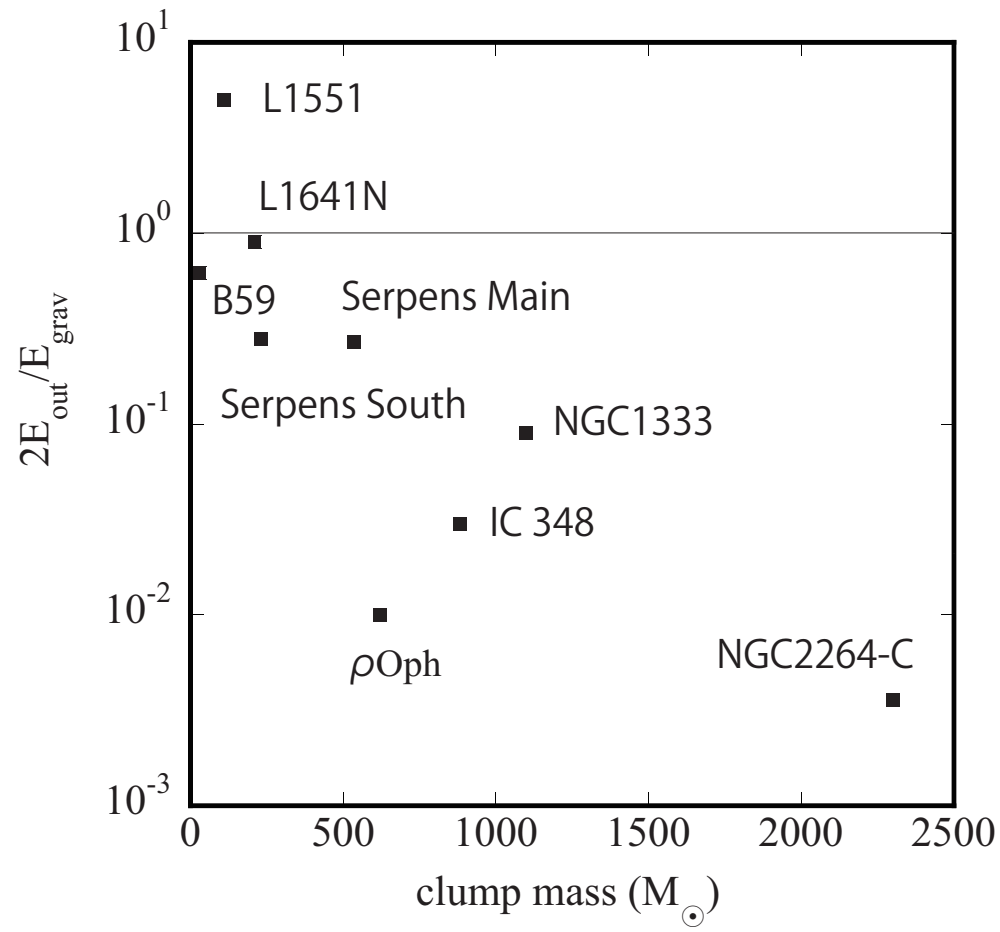

Fig. 2. Ratios of twice the outflow kinetic energy $2 E_{\text {out }}$ to the gravitational binding energy of the clump $E_{\text {grav }}$ for nearby cluster-forming clumps as a function of clump mass.

Krumholz, M. R., Bate, M. R., Arce, H. G., et al. 2014, in Protostars and Planets VI, H. Beuther, R. S. Klessen, C. P. Dullemond, and T. Henning (eds.), University of Arizona Press, Tucson, p. 243

Lada, C. J. 2010, Philosophical Transactions of the Royal Society A: Mathematical, Physical and Engineering Sciences, 368, p. 713

Mac Low, M.-M., \& Klessen, R. 2004, Reviews of Modern Physics, 76, p. 125

Maury, A. J., Andre, P., \&Li, Z.-Y., 2009, A\&A, 499, 175

Nakamura, F. \& Li, Z.-Y. 2007, ApJ, 783, 8

Nakamura, F. \& Li, Z.-Y. 2014, ApJ, 662, 395

Nakamura, F., Kamada, Y., Kamazaki, T. et al. 2011a, ApJ, 726, 46

Nakamura, F., Sugitani, K., Shimajiri, Y., et al. 2011b, ApJ, 737, 56

Nakamura, F., Miura, T., Kitamura, Y., et al. 2012, ApJ, 746, 25

Stojimirovic, I., Narayanan, G., Snell, R. L., \& Bally, J. 2006, ApJ, 649, 280

Sugitani, K., Nakamura, F., Tamura, M., et al. 2010, ApJ, 716, 299

Tan, J. C., Krumholz, M. R., \& McKee, C. F. 2006, ApJ, 641, 121 